Dr. J. D. Whitehead (University of Queensland) then gave a critical survey of the theories of formation of irregularities in the $F^{\prime}$ region. He pointed out that, for hydromagnetic waves, the percentage fluctuation of electron density is equal to the percentage fluctuation of magnetic field, and this appears to demand unreasonably large amplitudes for the waves. The instability theory was criticized on the grounds that the observed vertical motions of the $F$ region were not those required by the theory. Dr. Whitehead suggested that the irregularities were produced either by air movements associated with internal gravity waves, or by irregular electric fields, and the theories of formation of irregularities by these mechanisms were considered. Dr. Whitehead stressed the need for more detailed measurements of the sizes and shapes of the irregularities so that the predictions of the theories could be tested.

On the second day of the conference, satellite scintillations were discussed. Unlike a radio star, a satellite sweeps rapidly across the sky from horizon to horizon, and so the method is particularly valuable for examining the geographical distribution of irregularities, especially if observations from several stations are combined. Dr. G. H. Munro (Radio Research Board, Sydney) described observations of this type, in which transmissions on $20 \mathrm{Mc} / \mathrm{s}$ from the satellites Nora Alice $I$ and $I I$ were used. The results showed that irregularities tend to occur in patches which become more frequent, and larger, at higher latitudes. The patches appear to be elongated east-west and may stretch in this direction for 1,000 miles or more. From measurements of the times of onset of scintillations at spaced stations the heights of the patches may also be determined.

Mr. I. A. Parkin (University of Adelaide) discussed the theory of the diffraction process which links the irregularities in the ionosphere to the pattern of amplitude and phase which is observed over the ground. He considered both a source at infinity and at a finite distance. If detailed deductions about the sizes and shapes of the irregularities are to be made, it is necessary to use frequencies such that the fluctuation of phase across the emerging wavefront is less than one radian. It is then possible to relate the auto-correlation function of ampli- tude measured on the ground to the auto-correlation function of the irregular medium. Mr. Parkin also pointed out that at a given distance from the irregularities certain Fourier components should be absent in the amplitude pattern, and so measurements of the power spectrum of the pattern may enable the height of the irregularities to be determined. Some discussion of the relation between the diffraction approach and the method of geometrical optics followed Mr. Parkin's paper, and it was agreed that in principle the diffraction method is always valid, but in practice it is most useful when the phase deviation is small, while the method of geometrical optics is useful when the phase deviation is large.

Attention then turned to irregularities at lower heights. Dr. R. G. Roper (University of Adelaide) described measurements of winds and turbulence in the height range 70-110 km, and Dr. J. D. Whitehead (University of Queensland) discussed the formation of sporadic $E$ which, at temperate latitudes, he considered to be produced by vertical shears in the horizontal wind. However, the irregular wind shears described by Dr. Roper had the greatest magnitude at the equinoxes, and this does not agree with the occurrence of sporadic $E$, which is most prevalent in summer. Dr. Whitehead also discussed the formation of equatorial sporadic $E$, which he suggested was due to irregularities formed by sound waves.

The conference concluded with two papers of a more technical nature. Dr. L. H. Heisler (Radio Research Board, Sydney) described equipment for the investigation of the variation with frequency of the amplitude of radio waves reflected from the ionosphere, and Dr. J. D. Whitehead (University of Queensland) described a proposed method for measuring with increased precision the virtual height of reflexion.

During the conference the opportunity was taken to discuss observations of the forthcoming S66 Beacon satellite which are being planned in Australia and New Zealand. The general feeling was that much more work needs to be done before the mode of formation of ionospheric irregularities is properly understood, and that intensive observations using the Beacon satellite are likely to make a valuable contribution towards this end.

B. H. BrigGs

\title{
OXIDATIVE PHOSPHORYLATION AND RELATED PROCESSES
}

\section{$\mathrm{T}$} HE Medical Sciences Club of South Australia and the Australian Biochemical Society (South Australian Branch) held a symposium on "Oxidative Phosphorylation and Related Processes" in the Biochemistry Department, University of Adelaide, during June 14-15.

Prof. E. C. Slater (Amsterdam) opened the symposium with a review of present-day theories on the formation of adenosine triphosphate in mitochondria. He contrasted Mitchell's theory that this process is the reversal of hydrolysis of adenosine triphosphate by an anisotropic adenosine triphosphatase to the more widely accepted view that adenosine triphosphate is produced by reactions involving a number of specific intermediates formed at several different 'sites' in the sequence of oxidationreduction catalysts in mitochondria. Much of the evidence for such intermediates has been obtained in experiments with oligomycin, which prevents formation of adenosine triphosphate but does not inhibit other reactions such as the reduction of nicotinamide-adenine dinucleotide by succinate; for thermodynamic reasons, this reduction must be coupled to exergonic processes such as the decomposition of compounds like the proposed intermediates. However, these investigations do not allow a final choice between Mitchell's theory and that preferred by Slater, Chance and others involving specific intermediates of the type often formulated as $A \sim C$. It was pointed out in discussion that the existence of adenosine triphosphate and adenosine diphosphate in a region of the mitochondrion not accessible to oligomycin would invalidate certain conclusions drawn from experiments with this inhibitor.

Prof. J. S. Sturtevant (Yale) described kinetic investigations of the interaction of cytochrome $b_{2}$ (the $\mathrm{L}(+)$-lactate dehydrogenase of yeast) with lactate and ferricyanide. Using a stopped-flow spectrophotometer at a number of wave-lengths, evidence was obtained which indicated a preliminary reduction of flavin mononucleotide, an intra-molecular transfer of electrons to protohæm, and interaction of both prosthetic groups of this flavohæmoprotein with ferricyanide. The results support earlier suggestions by Morton, who found equimolar quantities of these prosthetic groups in the enzyme and proposed a mechanism involving intra-molecular transfer of electrons from flavin to hæm. The need to find if cytochrome $b_{2}$ is a constituent of yeast mitochondria was considered in the general discussion following these papers. Clearly a single flavohæmoprotein is a potential model for the more complex flavin-hæm systems of the whole mitochondrion.

Dr. M. R. Atkinson (Adelaide) reviewed recent evidence for phosphorylated and unphosphorylated intermediates of oxidative phosphorylation and described electrophoretic investigations (with urea-starch gels or on paper after 
tryptic hydrolysis) of the radioactive products obtained on exposure of mitochondria and mitochondrial extracts to radioactive orthophosphate. He proposed a model for adenosine triphosphate synthesis in a concerted reaction with adenosine diphosphate, orthophosphate and an activated derivative of cytochrome $c$ formed through acylation of the histidyl residue on the hæm iron by the adjacent glutamyl side-chain.

Dr. A. M. Snoswell (New South Wales) described experiments in which reduction of nicotinamide-adenine dinucleotide by succinate in mitochondria from rabbit heart was coupled to electron transfer from succinate or from tetramethylphenylenediamine to oxygen through cytochrome $c$ and cytochrome oxidase. The resistance of the coupled reductions to oligomycin was interpreted as evidence for activated intermediates other than adenosine triphosphate, which permit reduction of nicotinamide-adenine dinucleotide by compounds of higher oxidation-reduction potential. Dr. M. L. Birt (Melbourne) described related experiments with liver mitochondria prepared in a Krebs buffer in which reduction of the co-enzyme by succinate was inhibited by dinitrophenol but was not entirely inhibited by anaerobic conditions or by antimycin $A$ and amytal. The relative importance of reduction through enzymes such as malate dehydrogenase and reduction of coenzyme through reversal of the respiratory chain was discussed.

Prof. R. N. Robertson (Adelaide) reviewed recent investigations of the relationship of oxidative phosphorylation to the active transport of ions against an electrochemical gradient. Active transport need not be coupled to hydrolysis of adenosine triphosphate, but transport of ions or phosphorylation of adenosine diphosphate may be alternative consequences of the oxidative generation of compounds of the $A \sim B$ type or the oxidative separation of positive and negative charge, as envisaged. by Mitchell. Dr. J. Charnock (Adelaide) described experiments on an adenosine triphosphatase from the microsomal fraction of kidney; this enzyme requires sodium and potassium ions for full activity and is inhibited by ouabain. Dr. Charnock discussed the evidence that a phosphoprotein intermediate is involved in the action of the enzyme and that the phosphoprotein might carry cations across membranes. Dr. B. Hetzel (Adelaide) discussed the uncoupling of oxidative phosphorylation by thyroxine and triiodothyronine and reviewed evidence that this process was involved in the physiological action of these com. pounds.

In his concluding remarks, Prof. R. K. Morton (Adelaide), who arranged the meeting, emphasized the need for caution in interpreting the results of investigations with phosphorylated compounds derived from mitochondria; these might differ from the true intermediates, just as the isolation of phosphorylated enzymes does not prove the involvement of these compounds in enzymatic phosphoryl transfer.

The cryptic nature of many publications on oxidative phosphorylation caused much good-humoured but frustrated comment, and most participants looked forward to the publication of experimental details which will permit more detailed examination of many recent claims in this remarkably active field of scientific investigation.

M. R. Atrinson

[The late] R. K. Morton

\section{ORGANIC MATTER AND SOIL PRODUCTIVITY}

T has commonly been accepted that farmyard manure 1 is desirable or even essential to maintain or improve soil fertility, because it exerts a beneficial effect on the physical condition of practically all mineral soils and steadily supplies plant nutrients as it decomposes. Market gardeners have relied on stable and byre manures from the nearest towns and farmers in arable areas have kept cattle over winter to provide manure for the potato and root crops in the rotation. Most growers in fact have been so convinced of the value of regular additions of organic waste material to the soil that, in face of declining supplies of farmyard manure, they have adopted various methods of green manuring or of composting or even of bringing in peat. In effect, a normal dressing of 10-15 tons of farmyard manure amounts to some 2 tons of organic matter per acre or 0.2 per cent in the top 9 in. of soil. This amount is barely measurable by sampling and analysis. It is substantially the same as the annual leaf litter in woodland; it is not much more than the amount in the stubble and roots of a cereal crop; it is much less than what is incorporated in the soil by ploughing in grass turf. Nevertheless, there remains the conviction that on many types of soil fresh organic matter confers a benefit that is not obtained from plant nutrients alone.

It is practically impossible to make a strict comparison of organic manures with inorganic fertilizers since the former contain nutrients that become available to the plant over a more or less prolonged period and, at the same time, modify the soil in a manner that affects all the factors like æration, water movement and biological activity that play a part in crop development. There is undoubtedly evidence that the crop responses to the total nutrients in farmyard manure are very much less than those obtained from the same amounts of nutrients applied as soluble fertilizers. There have also been recent assessments from field experiments which indicate that the merit of farmyard manure is to be attributed almost solely to its nutrient content. But the fact remains that on some soils it is not possible to produce maximum crops, with or without fertilizers, unless farmyard manure has been used. It is not surprising, therefore, that investigators have been searching for more precise information on the effects of additions of organic matter to the soil. Is it possible, for example, that organic matter may affect the availability of nutrients to the plant, or may produce derivatives that influence plant growth either directly, through absorption by the roots, or indirectly, by controlling the incidence of disease?

These matters are the subject of a well-documented review by D. C. Whitehead entitled "Some Aspects of the Influence of Organic Matter on Soil Fertility" in a recent issue of Soils and Fertilizers (26, No. 4, Commonwealth Bureau of Soils; 1963). He discusses the question of soil nitrogen released from organic residues or fixed by symbiotic or free-liming organisms; of the ability of organic acids to release insoluble forms of phosphorus and potassium, and certain trace elements, from the soil particles. $\mathrm{He}$ also directs attention to the absorption by the roots of organic molecules that could influence plant metabolismthis covers such substances as amino-acids, vitamins, phenolic compounds and auxins-and to the ability of organic matter to favour saprophytic organisms rather than parasitic; there are also various decomposition products that are toxic to fungi. There is much contradictory evidence and obviously a need for further work to establish the significance of these compounds. They are known to be produced, but the quantities concerned and the probable interactions are still largely speculative.

Closely related to this review is an article on "The Biological Nature of Soil Productivity", by G. V. Jacks $(26$, No. $3 ; 1963)$. This is designed to show how fertility is dependent on bio-physical rather than on physicochemical changes in the soil. It is the action of plants that converts radiant energy into energy for animals and 